\title{
Antagonismes entre Levures pathogènes et quelques Bactéries
}

\author{
par Manuel ANSEL et Monique THIBAUT
}

Si la levure Candida albicans se trouve habituellement et avant tout dans le tube digestif, on peut considérer que plus exceptionnellement et apparemment Cryptococcus neoformans peut s'y trouver aussi, car il a été maintes fois isolé de divers aliments et l'on a signalé d'autre part quelques cas de cryptococcose uniquement digestive.

Nous avons voulu rechercher les antagonismes auxquels pouvaient être soumises ces deux levures pathogènes dans le tube digestif où elles subissent l'action non seulement des diastases digestives, mais aussi celle de nombreuses bactéries saprophytes ou pathogènes.

Dans cette étude préliminaire, nous n'avons étudié les biocoenoses bactéries-levures que du point de vue quantitatif et seulement dans le sens action des bactéries sur la multiplication de ces deux levures.

Notre choix s'est porté sur des bactéries qui se trouvent habituellement dans le tube digestif, telles que Escherichia coli ou qui y sont introduites pour des raisons thérapeutiques diverses. De ces dernières, nous avons retenu trois genres et six espèces :

- le genre Streptococcus avec les espèces Streptococcus lactis et Streptoccoccus thermophilus, lactis,

- le genre Lactobacillus avec les espèces Lactobacillus acidophilus, bulgaricus,

- et, enfin, un microbe sporulant, le Bacillus subtilis.

Pour la comparaison des effets entre les germes vivants et leurs produits de métabolisme, nous avons comparé avec l'action des bactéries indiquées, celle des produits du métabolisme de Lactobacterium bulgaricum.

\section{Technique utilisée :}

Cryptococcus neoformans et Candida albicans ont été ensemencés en une quantité égale d'une même suspension, dans des tubes de bouillon peptoné à $1 \%$, glycosé à $2 \%$ et lactosé à $2 \%$. En conservant des tubes témoins, nous avons dans les autres tubes introduit une suspension de bactéries lyophilisées, correspondant à la $1 / 200^{\circ}$ partie de la dose qui peut être administrée à l'homme adulte. Après culture à l'étuve à $37^{\circ}$, 
des prélèvements ont été faits après homogénéisation des bouillons, ceci après 14 ou 15 heures, puis après un temps trois fois supérieur : 42 et 45 heures. Ces laps de temps représentent les durées limites habituelles du transit du bol alimentaire dans le tube digestif de l'homme.

Les numérations ont été faites à l'hématimètre de Malassez.

Les bactéries, utilisées sous forme lyophilisée, ont été étudiées :

- soit séparément, tels Bacillus subtilis, Escherichia coli, Streptococcus lactis, Lactobacterium acidophilum ;

- soit en associations, dont l'une comprenait: Streptococcus lactis et les Lactobacterium acidophilum et bulgaricum; l'autre deux Streptococcaeae: Streptococcus lactis et thermophilus et deux Lactobacteriaceae : Lactobacterium acidophilum et lactis.

\section{Résultats :}

Par dixième de millimètre cube, les nombres des levures après 14 et 42 heures pour Cryptococcus neoformans, 15 et 45 heures pour Candida albicans, ont été les suivants:

\section{Cryptococcus neoformans.}

Par ordre d'activité.

$1^{\circ}$ Après 14 heures.

Témoin : 370 éléments par dixième de millimètre cube.

Association trispécifique : 361 .

Lactobacterium acidophilum : 342 .

Escherichia coli variété communis : 308.

Produits du métabolisme de Lactobacterium bulgaricum : 304.

Association tétraspécifique : 282.

Bacillus subtilis : 250.

Streptococcus lactis : 231.

\section{$2^{\circ}$ Après 42 heures.}

Témoin : 693 éléments par dixième de millimètre cube.

Escherichia coli variété communis : 640 .

Seuls produits du métabolisme de Lactobacterium bulgaricum : 585 .

Association tétraspécifique : 367.

Association trispécifique : 364 .

Lactobacterium acidophilum : 321.

Streptococcus lactis : 237.

Bacillus subtilis : 236.

\section{Candida albicans.}

Par ordre d'activité. 
$1^{\circ}$ Après 15 heures.

Témoin : 865 éléments par dixième de millimètre cube.

Produits du métabolisme de Lactobacterium bulgaricum : 820 .

Association tétraspécifique : 763.

Escherichia coli variété communis : 702.

Association trispécifique : 394 .

Lactobacterium acidophilum : 258.

Streptococcus lactis : 223.

Bacillus subtilis : 132.

$2^{\circ}$ Après 45 heures.

Témoin : 1.315 éléments par dixième de millimètre cube.

Escherichia coli variété communis : 1.426.

Association tétraspécifique : 1.054 .

Produits du métabolisme de Lactobacterium bulgaricum : 854.

Association trispécifique : 824 .

Lactobacterium acidophilum : 585.

Streptococcus lactis : 549.

Bacillus subtilis : 167.

\section{Interprétation des résultats}

I. Pour Cryptococcus neoformans, on constate :

$1^{\circ}$ Une accoutumance de cette levure vis-à-vis : d'une part du colibacille : Escherichia coli, car, après 42 heures de culture, le nombre des cryptocoques présents avec l'Escherichia est à peu près le même que celui des cryptocoques cultivés seuls ; d'autre part, une accoutumance vis-à-vis des produits du métabolisme du Lactobacterium bulgaricum: le nombre des cryptocoques a presque doublé.

$2^{\circ}$ Une simple action freinatrice sur la multiplication, des quatre bactéries associées, d'où est absente l'espèce $L$. bulgaricum .

$3^{\circ}$ Une action nettement zymostatique des trois bactéries associées où il n'y a pas le Streptococcus thermophilus, mais où se trouve par contre Lactobacterium bulgaricum, remplaçant le Lactobacterium lactis'; et une action nettement zymostatique aussi du seul Streptococcus lactis.

$4^{\circ}$ Une action progressivement et légèrement zymolytique du Lactobacterium acidophilum et du Bacillus subtilis.

\section{Pour Candida albicans.}

$1^{\circ}$ Il y a non seulement un effet d'accoutumance du Candida vis-à-vis de l'Escherichia coli, mais il paraît vraisemblable que le colibacille favorise même la multiplication du Candida albicans, le nombre de Candida en culture avec Escherichia est plus élevé que dans les tubes où il n'y a que le Candida. 
$2^{\circ}$ Bien qu'elles freinent nettement la croissance du Candida, l'ensemble des bactéries lactiques ne peuvent empêcher que la multiplication ne continue. Après 45 heures, le nombre de Candida en culture avec ces lactobactéries a plus que doublé, bien que nettement inférieur, de la moitié en moyenne, au tube témoin.

$3^{\circ}$ Nous ne trouvons, vis-à-vis de Candida albicans, d'action zymostatique constante que dons la biocenose avec Bacillus subtilis.

\section{Conclusions}

$1^{\circ}$ Nous pensons qu'il n'y a aucune action bénéfique à attendre de l'Escherichia coli par suite de l'accoutumance du Cryptococcus à son égard, mais aussi surtout par suite de son action favorisante même sur la multiplication du Candida albicans.

$2^{\circ}$ Dans l'ensemble, les bactéries lactiques ont une action heureuse sur la multiplication des levures Cryptococcus neoformans ou Candida albicans, qui est nettement freinée. Leur utilisation dans la prévention des candidoses ou éventuellement des cryptococcoses digestives est justifiée. Elles pourraient même être utilisées comme adjuvants valables dans les infections déclarées du tube digestif par ces levures.

$3^{\circ}$ Il paraît très clairement que les bactéries lactiques prises isolément, notamment Streptococcus lactis et Lactobacterium acidophilum ont une action plus énergique seules, que lorsqu'elles sont associées entre elles.

$4^{\circ}$ Le Bacillus subtilis, dans tous les cas, a toujours manifesté une action nette et forte. Il est zymostatique, peut-être même légèrement zymolytique.

$5^{\circ}$ Le mécanisme d'action de ces bactéries est difficile à préciser. L'effet compétitif vis-à-vis des sucres, les variations du $\mathrm{pH}$ paraissent cependant moins importants que l'action probable d'antibiotiques. Le grand nombre d'antibiotiques produits par Bacillus subtilis semble indiquer que ceux-ci sont les agents antilevures produits par ces bactéries, et la nisine extraite à partir du Streptococcus lactis expliquerait aussi l'action particulièrement remarquable de cette bactérie prise isolément.

\section{Résumé}

La présence de Candida albicans et parfois de Cryptococcus neoformans dans les voies digestives, nous a incités à chercher quelle influence peuvent avoir sur ces deux levures divers microbes qui se trouvent aussi dans le tube digestif soit spontanément, soit après introduction pour des raisons thérapeutiques.

Nous avons recherché l'effet quantitatif sur la croissance et la multiplication des levures par numération de celles-ci dans la culture en biocoenose avec les bactéries suivantes: Escherichia coli, les Streptococcus lactis et Str. thermophilus, les Lactobacillus acidophilus, L. bulgaricus et $L$. lactis et enfin avec Bacillus subtilis. Le nombre des levures par un dixième de millimètre cube a été compté après 14 et 42 ou 15 et 45 heures, ce qui représente les durées extrêmes de transit du bol alimentaire chez 


\begin{tabular}{|c|c|}
\hline 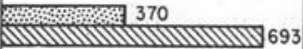 & Tem. \\
\hline काप 308 & Ec. \\
\hline 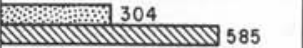 & MetLb. \\
\hline 282 & $S \ell, t+L a, \ell$ \\
\hline 361 & $S l+L a, b$ \\
\hline $\begin{array}{ll} & \\
342 & \end{array}$ & La. \\
\hline 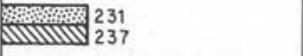 & sl. \\
\hline $\begin{array}{l}\text { Mingsing } 250 \\
236\end{array}$ & Bs. \\
\hline
\end{tabular}

\section{TABLEAU I}

Nombre de cellules de Cryptococcus neoformans par dixième de millimètre cube après 14 heures ( $1^{\text {re }}$ colonne en pointillé) et 42 heures ( $2^{e}$ colonne hachurée) dans une culture où elles sont ensemencées seules (Témoin) ou avec les bactéries indiquées.

\section{TABLEAU II}

Nombre de cellules de Candida albicans par dixième de millimètre cube après 15 heures $\left(1^{\text {ro }}\right.$ colonne pointillée) et 45 heures ( $2^{\mathrm{e}}$ colonne hachurée) dans une culture où elles ont été ensemencées seules (Témoin) ou avec les bactéries indiquées.

\begin{tabular}{|c|c|}
\hline 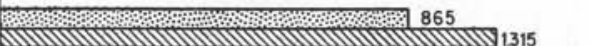 & Tem. \\
\hline 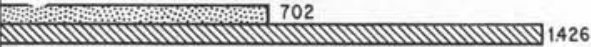 & Ec. \\
\hline 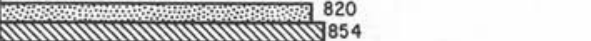 & Met Lb. \\
\hline 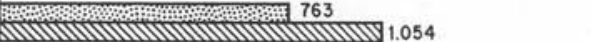 & $S \ell, t+L a, l$ \\
\hline 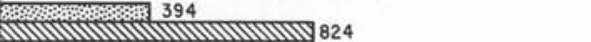 & $S \ell+L a, b$ \\
\hline समापू 258 & La \\
\hline 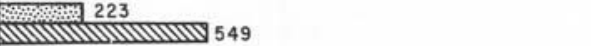 & sl. \\
\hline $\begin{array}{l}132 \\
1201\end{array}$ & Bs. \\
\hline
\end{tabular}

Dans les deux tableaux, les abréviations suivantes indiquent: Tém. : Témoin; Met L b : seuls produits du métabolisme de Lactobacterium bulgaricum; $\mathrm{S} 1+\mathrm{L}$ a b : les trois bactéries associées Streptococcus lactis, Lactobacterium acidophilum et Lactobacterium bulgaricum; $\mathrm{S} 1 \mathrm{t}+\mathrm{L}$ a 1: l'association Streptococcus lactis et Streptococcus thermophilus avec Lactobacterium acidophilum et $L$. lactis; E c : Escherichia coli; $\mathrm{L}$ a : Lactobacterium acidophilum; S1: Streptococcus lactis seul. 
l'homme. Nou avons constaté que le Cryptococcus neoformans comme aussi le Candida albicans s'accoutument à la présence de l'Escherichia coli et que ce dernier favorise même la multiplication du Candida albicans. Les bactéries lactiques étudiées, soit du genre Streptococcus, soit du genre Lactobacterium abaissent nettement le taux de multiplication des levures précitées. Leur utilisation dans la prévention des mycoses du tube digestif par Cryptococcus neoformans ou Candida albicans est donc non seulement justifiée, mais elle doit même être recommandée dans la prévention des accidents à levures au cours des traitements par les antibiotiques ou les corticoïdes.

Les bactéries lactiques, notamment Streptococcus lactis et Lactobacterium acidophilus sont plus actives quand elles sont prises isolément que lorsqu'elles sont associées à plusieurs espèces voisines. Le Bacillus subtilis manifeste une activité au moins égale à celle des deux bactéries précitées, quelquefois plus forte puisqu'il peut même avoir une légère action zymolytique.

Le mécanisme d'action n'a pas été étudié. L'effet compétitif vis-à-vis des sucres, les variations du $\mathrm{pH}$ paraissent cependant moins importants que l'action probable d'antibiotiques déjà connus secrétés par Bacillus subtilis ou par Streptococcus lactis (Nisine).

Travail du Service de Mycologie de l'Institut de Parasitologie de la Faculté de Médecine de Paris (Directeur Professeur : L.-C. BRUMPT) 